\title{
Article
}

\section{Conditional Intuitionistic Fuzzy Mean Value}

\author{
Katarína Čunderlíková (D)
}

check for

updates

Citation: Čunderlíková, K Conditional Intuitionistic Fuzzy Mean Value. Axioms 2021, 10, 97. https://doi.org/10.3390 /axioms10020097

Academic Editor: Radko Mesiar

Received: 19 April 2021

Accepted: 18 May 2021

Published: 21 May 2021

Publisher's Note: MDPI stays neutral with regard to jurisdictional claims in published maps and institutional affiliations.

Copyright: (C) 2021 by the author. Licensee MDPI, Basel, Switzerland. This article is an open access article distributed under the terms and conditions of the Creative Commons Attribution (CC BY) license (https:/ / creativecommons.org/licenses/by/ $4.0 /)$.
Mathematical Institute, Slovak Academy of Sciences, Štefánikova 49, 81473 Bratislava, Slovakia; cunderlikova.lendelova@gmail.com

\begin{abstract}
The conditional mean value has applications in regression analysis and in financial mathematics, because they are used in it. We can find papers from recent years that use the conditional mean value in fuzzy cases. As the intuitionstic fuzzy sets are an extension of fuzzy sets, we will try to define a conditional mean value for the intuitionistic fuzzy case. The conditional mean value in crisp intuitionistic fuzzy events was first studied by V. Valenčáková in 2009. She used Gödel connectives. Her approach can only be used for special cases of intuitionistic fuzzy events, therefore, we want to define a conditional mean value for all elements of a family of intuitionistic fuzzy events. In this paper, we define the conditional mean value for intuitionistic fuzzy events using Lukasiewicz connectives. We use a Kolmogorov approach and the notions from a classical probability theory for construction. B. Riečan formulated a conditional intuitionistic fuzzy probability for intuitionistic fuzzy events using an intuitionistic fuzzy state in 2012. In classical cases, there exists a connection between the conditional probability and the conditional mean value, therefore we show a connection between the conditional intuitionistic fuzzy probability induced by the intuitionistic fuzzy state and the conditional intuitionistic fuzzy mean value.
\end{abstract}

Keywords: intuitionistic fuzzy event; intuitionistic fuzzy observable; intuitionistic fuzzy state; product; conditional intuitionistic fuzzy probability; conditional intuitionistic fuzzy mean value

MSC: 03B52; 60A86; 60A10

\section{Introduction}

In general, a conditional mean value has many applications in regression analysis and in financial mathematics and insurance. The most used notion in these areas is uncertainty. The notion of uncertainty has two aspects. The first one is understood as risk uncertainty and it is modeled by a stochastic apparatus. The second one is vagueness, which can be modeled by fuzzy methodology.

In [1], A. de Korvin and R. Kleyle studied a conditional expectation in a fuzzy case and they showed its use for Gaussian distribution. A conditional variance of fuzzy random variables needs for its definition the notion of a conditional mean value (see [2]). An approach to modeling risk by the conditional value at risk methodology under imprecise and soft conditions was solved in [3]. In [4], C. You discussed the properties of a conditional mean value for fuzzy variables such as the Hölders inequality. In [5], M. Bertanha and G. W. Imbens showed the use of a conditional mean value for testing an external validity in fuzzy regression discontinuity designs. B. Riečan and M. Jurečková studied a notion of conditional expectation of observables on MV-algebras of fuzzy sets and on probability MV-algebras with product (see [6,7]). The intuitionistic fuzzy sets introduced by K. T. Atanassov in [8] are a generalization of Zadeh's fuzzy sets in [9,10], given by $(f, 1-f)$, where $f$ is a fuzzy set. As there are known practical applications in classical cases and fuzzy cases, it is interesting to study a notion of conditional expectation in a family of intuitionistic fuzzy sets. 
In [11], V. Valenčáková defined a conditional mean value $E(x \mid y)$ for crisp intuitionistic fuzzy events $\mathcal{A}=\left\{\left(\chi_{A}, 1-\chi_{A}\right)\right\} \subset \mathcal{F}=\left\{\left(\mu_{A}, v_{A}\right) ; \mu_{A}+v_{A} \leq 1_{\Omega}\right\}$ as a Borel function $g: R \rightarrow R$ satisfying the following equality

$$
E\left(x_{y(C)}\right)=\int_{C} g d m_{y}
$$

for every $C \in \mathcal{B}(R)$ and $y(C) \in \mathcal{A}$. There, $y: \mathcal{B}(R) \rightarrow \mathcal{A}, x: \mathcal{B}(R) \rightarrow \mathcal{F}$ are the M-observables and $m: \mathcal{F} \rightarrow[0,1]$ is an M-state. She used the Gödel connectives $\vee \wedge$ given by

$$
\mathbf{A} \vee \mathbf{B}=\left(\mu_{A} \vee \mu_{B}, v_{A} \wedge v_{B}\right), \mathbf{A} \wedge \mathbf{B}=\left(\mu_{A} \wedge \mu_{B}, v_{A} \vee v_{B}\right),
$$

for any $\mathbf{A}, \mathbf{B} \in \mathcal{F}$.

In this paper, we define a conditional mean value for the family of intuitionistic fuzzy events $\mathcal{F}$. We use the Lukasiewicz connectives $\oplus, \odot$ given by

$$
\begin{aligned}
& \left.\mathbf{A} \oplus \mathbf{B}=\left(\left(\mu_{A}+\mu_{B}\right) \wedge 1_{\Omega},\left(v_{A}+v_{B}-1_{\Omega}\right) \vee 0_{\Omega}\right)\right), \\
& \left.\mathbf{A} \odot \mathbf{B}=\left(\left(\mu_{A}+\mu_{B}-1_{\Omega}\right) \vee 0_{\Omega},\left(v_{A}+v_{B}\right) \wedge 1_{\Omega}\right)\right),
\end{aligned}
$$

for any $\mathbf{A}, \mathbf{B} \in \mathcal{F}$. We show the connection with a conditional intuitionistic probability $\mathbf{p}(\mathbf{A} \mid x)$ introduced by B. Riečan in [12] as a Borel measurable function $f$ (i.e., $\left.B \in \mathcal{B}(R) \Longrightarrow f^{-1}(B) \in \mathcal{B}(R)\right)$ such that

$$
\int_{B} \mathbf{p}(\mathbf{A} \mid x) d \mathbf{m}_{x}=\mathbf{m}(\mathbf{A} \cdot x(B))
$$

for each $B \in \mathcal{B}(R)$, where $\mathbf{m}: \mathcal{F} \rightarrow[0,1]$ is the intuitionistic fuzzy state, $\mathbf{A} \in \mathcal{F}$ is an intuitionistic fuzzy event and $x: \mathcal{B}(R) \rightarrow \mathcal{F}$ is an intuitionistic fuzzy observable. This conditional intuitionistic fuzzy probability is induced by an intuitionistic fuzzy state.

The paper is organized as follows: Section 2 includes the basic notions from intuitionistic fuzzy probability theory as an intuitionistic fuzzy event, an intuitionistic fuzzy state, an intuitionistic fuzzy observable and an intuitionistic fuzzy mean value. In Section 3, we present the main results of the research. First, we formulate a definition of an indefinite integral. Then we define a conditional intuitionistic fuzzy mean value for the intuitionistic fuzzy events. Next, we show a connection with a conditional intuitionistic probability induced by an intuitionistic fuzzy state. The last section contains concluding remarks and the direction of future research.

We note that in the whole text we use the notation IF as an abbreviation for 'intuitionistic fuzzy.'

\section{Basic Notions of the Intuitionistic Fuzzy Probability Theory}

In this section, we recall the definitions of basic notions connected with intuitionistic fuzzy probability theory (see [13-15]).

Definition 1 ([13-15]). Let $\Omega$ be a nonempty set. An IF-set $\mathbf{A}$ on $\Omega$ is a pair $\left(\mu_{A}, v_{A}\right)$ of mappings $\mu_{A}, v_{A}: \Omega \rightarrow[0,1]$ such that $\mu_{A}+v_{A} \leq 1_{\Omega}$.

Definition 2 ([13-15]). Start with a measurable space $(\Omega, \mathcal{S})$. Hence, $\mathcal{S}$ is a $\sigma$-algebra of subsets of $\Omega$. An IF-event is called an IF-set $\mathbf{A}=\left(\mu_{A}, v_{A}\right)$ such that $\mu_{A}, v_{A}: \Omega \rightarrow[0,1]$ are $\mathcal{S}$-measurable.

The family of all $I F$-events on $(\Omega, \mathcal{S})$ will be denoted by $\mathcal{F}, \mu_{A}: \Omega \longrightarrow[0,1]$ will be called the membership function, $v_{A}: \Omega \longrightarrow[0,1]$ will be called the non-membership function.

If $\mathbf{A}=\left(\mu_{A}, v_{A}\right) \in \mathcal{F}, \mathbf{B}=\left(\mu_{B}, v_{B}\right) \in \mathcal{F}$, then we define the Lukasiewicz binary operations $\oplus, \odot$ on $\mathcal{F}$ by 


$$
\begin{aligned}
& \left.\mathbf{A} \oplus \mathbf{B}=\left(\left(\mu_{A}+\mu_{B}\right) \wedge 1_{\Omega},\left(v_{A}+v_{B}-1_{\Omega}\right) \vee 0_{\Omega}\right)\right), \\
& \left.\mathbf{A} \odot \mathbf{B}=\left(\left(\mu_{A}+\mu_{B}-1_{\Omega}\right) \vee 0_{\Omega},\left(v_{A}+v_{B}\right) \wedge 1_{\Omega}\right)\right)
\end{aligned}
$$

and the partial ordering is given by

$$
\mathbf{A} \leq \mathbf{B} \Longleftrightarrow \mu_{A} \leq \mu_{B}, v_{A} \geq v_{B} .
$$

In the IF-probability theory ([12]), instead of the notion of probability, we use the notion of state.

Definition 3 ([12]). Let $\mathcal{F}$ be the family of all IF-events in $\Omega$. A mapping $\mathbf{m}: \mathcal{F} \rightarrow[0,1]$ is called an IF-state, if the following conditions are satisfied:

(i) $\mathbf{m}\left(\left(1_{\Omega}, 0_{\Omega}\right)\right)=1, \mathbf{m}\left(\left(0_{\Omega}, 1_{\Omega}\right)\right)=0$;

(ii) if $\mathbf{A} \odot \mathbf{B}=\left(0_{\Omega}, 1_{\Omega}\right)$ and $\mathbf{A}, \mathbf{B} \in \mathcal{F}$, then $\mathbf{m}(\mathbf{A} \oplus \mathbf{B})=\mathbf{m}(\mathbf{A})+\mathbf{m}(\mathbf{B})$;

(iii) if $\mathbf{A}_{n} \nearrow \mathbf{A}$ (i.e., $\left.\mu_{A_{n}} \nearrow \mu_{A}, v_{A_{n}} \searrow v_{A}\right)$, then $\mathbf{m}\left(\mathbf{A}_{n}\right) \nearrow \mathbf{m}(\mathbf{A})$.

The third basic notion in the probability theory is the notion of an observable. Let $\mathcal{J}$ be the family of all intervals in $R$ of the form

$$
[a, b)=\{x \in R: a \leq x<b\} .
$$

Then the $\sigma$-algebra $\sigma(\mathcal{J})$ is denoted $\mathcal{B}(R)$ and it is called the $\sigma$-algebra of Borel sets and its elements are called Borel sets.

Definition 4 ([12]). By an IF-observable on $\mathcal{F}$ we understand each mapping $x: \mathcal{B}(R) \rightarrow \mathcal{F}$, satisfying the following conditions:

(i) $x(R)=\left(1_{\Omega}, 0_{\Omega}\right), x(\varnothing)=\left(0_{\Omega}, 1_{\Omega}\right)$;

(ii) if $A \cap B=\varnothing$, then $x(A) \odot x(B)=\left(0_{\Omega}, 1_{\Omega}\right)$ and $x(A \cup B)=x(A) \oplus x(B)$;

(iii) if $A_{n} \nearrow A$, then $x\left(A_{n}\right) \nearrow x(A)$.

If we denote $x(A)=\left(x^{b}(A), 1_{\Omega}-x^{\sharp}(A)\right)$ for each $A \in \mathcal{B}(R)$, then $x^{b}, x^{\sharp}: \mathcal{B}(R) \rightarrow \mathcal{T}$ are observables, where $\mathcal{T}=\{f: \Omega \rightarrow[0,1] ; f$ is $\mathcal{S}$-measurable $\}$.

Similar to the classical case, the following theorem can be proved $([12,16])$.

Theorem 1 ([16]). Let $x: \mathcal{B}(R) \longrightarrow \mathcal{F}$ be an IF-observable, $\mathbf{m}: \mathcal{F} \longrightarrow[0,1]$ be an IF-state. Define the mapping $\mathbf{m}_{x}: \mathcal{B}(R) \longrightarrow[0,1]$ by the formula

$$
\mathbf{m}_{x}(C)=\mathbf{m}(x(C)) .
$$

Then $\mathbf{m}_{x}: \mathcal{B}(R) \longrightarrow[0,1]$ is a probability measure.

Proof. In [16] Proposition 3.1.

In [17] we introduced the notion of product operation on the family of $I F$-events $\mathcal{F}$ and showed an example of this operation.

Definition 5 ([17]). We say that a binary operation $\cdot$ on $\mathcal{F}$ is a product if it satisfies the following conditions:

(i) $\left(1_{\Omega}, 0_{\Omega}\right) \cdot\left(a_{1}, a_{2}\right)=\left(a_{1}, a_{2}\right)$ for each $\left(a_{1}, a_{2}\right) \in \mathcal{F}$;

(ii) the operation - is commutative and associative;

(iii) if $\left(a_{1}, a_{2}\right) \odot\left(b_{1}, b_{2}\right)=\left(0_{\Omega}, 1_{\Omega}\right)$ and $\left(a_{1}, a_{2}\right),\left(b_{1}, b_{2}\right) \in \mathcal{F}$, then

$$
\left(c_{1}, c_{2}\right) \cdot\left(\left(a_{1}, a_{2}\right) \oplus\left(b_{1}, b_{2}\right)\right)=\left(\left(c_{1}, c_{2}\right) \cdot\left(a_{1}, a_{2}\right)\right) \oplus\left(\left(c_{1}, c_{2}\right) \cdot\left(b_{1}, b_{2}\right)\right)
$$


and

$$
\left(\left(c_{1}, c_{2}\right) \cdot\left(a_{1}, a_{2}\right)\right) \odot\left(\left(c_{1}, c_{2}\right) \cdot\left(b_{1}, b_{2}\right)\right)=\left(0_{\Omega}, 1_{\Omega}\right)
$$

for each $\left(c_{1}, c_{2}\right) \in \mathcal{F}$;

(iv) if $\left(a_{1 n}, a_{2 n}\right) \searrow\left(0_{\Omega}, 1_{\Omega}\right),\left(b_{1 n}, b_{2 n}\right) \searrow\left(0_{\Omega}, 1_{\Omega}\right)$ and $\left(a_{1 n}, a_{2 n}\right),\left(b_{1 n}, b_{2 n}\right) \in \mathcal{F}$, then $\left(a_{1 n}, a_{2 n}\right) \cdot\left(b_{1 n}, b_{2 n}\right) \searrow\left(0_{\Omega}, 1_{\Omega}\right)$

The following theorem provides an example of product operation for $I F$-events.

Theorem 2 ([17]). The operation · defined by

$$
\left(x_{1}, y_{1}\right) \cdot\left(x_{2}, y_{2}\right)=\left(x_{1} \cdot x_{2}, y_{1}+y_{2}-y_{1} \cdot y_{2}\right)
$$

for each $\left(x_{1}, y_{1}\right),\left(x_{2}, y_{2}\right) \in \mathcal{F}$ is a product operation on $\mathcal{F}$.

Proof. In [17] Theorem 1.

Since now $\mathbf{m}_{x}: \mathcal{B}(R) \rightarrow[0,1]$ plays an analogous role as $P_{\xi}: \mathcal{B}(R) \rightarrow[0,1]$, we can define IF-mean value $\mathbf{E}(x)$ by the same formula (see [18]).

Definition 6 ([18]). We say that an IF-observable $x$ is an integrable IF-observable, if the integral $\int_{R} t d \mathbf{m}_{x}(t)$ exists. In this case we define the IF-mean value

$$
\mathbf{E}(x)=\int_{R} t d \mathbf{m}_{x}(t)
$$

If the integral $\int_{R} t^{2} d \mathbf{m}_{x}(t)$ exists, then we define IF-dispersion $\mathbf{D}^{2}(x)$ by the formula

$$
\mathbf{D}^{2}(x)=\int_{R} t^{2} d \mathbf{m}_{x}(t)-(\mathbf{E}(x))^{2}=\int_{R}(t-\mathbf{E}(x))^{2} d \mathbf{m}_{x}(t)
$$

\section{Conditional Intuitionistic Fuzzy Mean Value}

In this section, we present the main results. First, we introduce our motivation from classical probability space.

In the classical probability space $(\Omega, \mathcal{S}, P)$ if $\xi, \eta$ are two random variables, then the conditional mean value $E(\xi \mid \eta)$ of $\xi$ with respect to $\eta$ can be defined as a Borel function $g: R \rightarrow R$ such that

$$
\int_{\eta^{-1}(B)} \xi d P=\int_{B} g d P_{\eta}
$$

for each $B \in \mathcal{B}(R)$. Here $P_{\eta}=P \circ \eta^{-1}$ is the probability distribution of $\eta$. In our case this idea could also be realised:

$$
\int_{x(B)} y d \mathbf{m}=\int_{B} f d \mathbf{m}_{x}
$$

Of course, first we must define $\int_{C} y d \mathbf{m}$, because we have defined $\int_{R} y d \mathbf{m}=\mathbf{E}(y)=$ $\int_{R} t d \mathbf{m}_{y}(t)$ only.

Definition 7. If $x, y: \mathcal{B}(R) \rightarrow \mathcal{F}$ are the IF-observable and $B \in \mathcal{B}(R)$ is fixed, then we define $y_{x(B)}: \mathcal{B}(R) \rightarrow \mathcal{F}$ by the formula

$$
y_{x(B)}(D)= \begin{cases}\left(0_{\Omega}, 1_{\Omega}\right), & \text { if } D=\varnothing \\ y(D \backslash\{0\}) \cdot x(B), & \text { if } D \neq R, D \in \mathcal{B}(R) \\ \left(1_{\Omega}, 0_{\Omega}\right), & \text { if } D=R\end{cases}
$$


Proposition 1. The mapping $y_{x(B)}$ is an IF-observable. If the IF-observable $y$ is integrable, then the IF-observable $y_{x(B)}$ is also integrable.

Proof. Let $C, D \in \mathcal{B}(R), C \cap D=\varnothing$. If $0 \notin C, 0 \notin D$, then using Definitions 4 and 5 we have

$$
\begin{aligned}
y_{x(B)}(C \cup D) & =y((C \cup D) \backslash\{0\}) \cdot x(B)=y(C \cup D) \cdot x(B)=(y(C) \oplus y(D)) \cdot x(B)= \\
& =y(C) \cdot x(B) \oplus y(D) \cdot x(B)=y(C \backslash\{0\}) \cdot x(B) \oplus y(D \backslash\{0\}) \cdot x(B)= \\
& =y_{x(B)}(C) \oplus y_{x(B)}(D) .
\end{aligned}
$$

If $0 \in C, 0 \notin D$, then $0 \in C \cup D$ and using Definition 4 and Definition 5, we obtain

$$
\begin{aligned}
y_{x(B)}(C \cup D) & =y((C \cup D) \backslash\{0\}) \cdot x(B)=y(C \backslash\{0\} \cup D) \cdot x(B)= \\
& =(y(C \backslash\{0\}) \oplus y(D)) \cdot x(B)=(y(C \backslash\{0\}) \cdot x(B)) \oplus(y(D) \cdot x(B))= \\
& =(y(C \backslash\{0\}) \cdot x(B)) \oplus(y(D \backslash\{0\}) \cdot x(B))=y_{x(B)}(C) \oplus y_{x(B)}(D) .
\end{aligned}
$$

If $0 \notin C, 0 \in D$, then $0 \in C \cup D$ and we have $y_{x(B)}(C \cup D)=y_{x(B)}(C) \oplus y_{x(B)}(D)$, similar to the previous case.

Since $y(C) \odot y(D)=\left(0_{\Omega}, 1_{\Omega}\right)$, then using Definition 5 we have

$$
y_{x(B)}(C) \odot y_{x(B)}(D)=(y(C \backslash\{0\}) \cdot x(B)) \odot(y(D \backslash\{0\}) \cdot x(B))=\left(0_{\Omega}, 1_{\Omega}\right) .
$$

If $A_{n} \nearrow A$, then

$$
y_{x(B)}\left(A_{n}\right)=y\left(A_{n} \backslash\{0\}\right) \cdot x(B) \nearrow y(A \backslash\{0\}) \cdot x(B)=y_{x(B)}(A) .
$$

Let $y$ be integrable, that is, there exists $\mathbf{E}(y)=\int_{R} t d \mathbf{m}_{y}(t)$. We want to prove that $y_{x(B)}$ is integrable, too. It suffices to prove that there exist the integrals

$$
\int_{[0, \infty)} t d \mathbf{m}_{y_{x(B)}}(t), \int_{(-\infty, 0)} t d \mathbf{m}_{y_{x(B)}}(t)
$$

Define the measure $\mu: \mathcal{B}(R) \rightarrow R$ by the formula

$$
\mu(D)=\mathbf{m}_{y_{x(B)}}(D)=\mathbf{m}(y(D \backslash\{0\}) \cdot x(B)) .
$$

Then $\mu$ is a measure and

$$
\mu(D)=\mathbf{m}(y(D \backslash\{0\}) \cdot x(B)) \leq \mathbf{m}(y(D) \cdot x(B)) \leq \mathbf{m}\left(y(D) \cdot\left(1_{\Omega}, 0_{\Omega}\right)\right)=\mathbf{m}(y(D)) .
$$

It follows,

$$
\begin{aligned}
0 \leq \int_{[0, \infty)} t d \mathbf{m}_{y_{x(B)}}(t) & =\int_{\{0\}} t d \mathbf{m}_{y_{x(B)}}(t)+\int_{(0, \infty)} t d \mathbf{m}_{y_{x(B)}}(t)=\int_{(0, \infty)} t d \mu(t) \leq \\
& \leq \int_{(0, \infty)} t d \mathbf{m}_{y}(t)<\infty
\end{aligned}
$$

On the other hand, for $t<0$ we have

$$
\int_{(-\infty, 0)} t d \mathbf{m}_{y_{x(B)}}(t)=\int_{(-\infty, 0)} t d \mu(t) \geq \int_{(-\infty, 0)} t d \mathbf{m}_{y}(t)>-\infty .
$$


Definition 8. If $x, y: \mathcal{B}(R) \rightarrow R$ are the IF-observables, such that $y$ is integrable, (i.e., there exists $\left.\mathbf{E}(y)=\int_{R} t d \mathbf{m}_{y}(t)\right)$, then the indefinite integral is defined by the formula

$$
\mathbf{E}\left(y_{x(B)}\right)=\int_{R} t d \mathbf{m}_{y_{x(B)}}(t)
$$

for fixed $B \in \mathcal{B}(R)$.

Proposition 2. Let $x, y: \mathcal{B}(R) \rightarrow R$ be the IF-observables and $y$ be integrable. Then $\mathbf{E}\left(y_{x(B)}\right)$ is a finite generalized measure.

Proof. Let $B=\bigcup_{i=1}^{\infty} B_{i}, B_{i}$ be disjoint. Then $x(B)=\bigoplus_{i=1}^{\infty} x\left(B_{i}\right) \leq\left(1_{\Omega}, 0_{\Omega}\right)$. Put

$$
\begin{aligned}
\mu(D) & =\mathbf{m}_{y_{x(B)}}(D)=\mathbf{m}(y(D \backslash\{0\}) \cdot x(B)), \\
\mu_{i}(D) & =\mathbf{m}_{y_{x\left(B_{i}\right)}}(D)=\mathbf{m}\left(y(D \backslash\{0\}) \cdot x\left(B_{i}\right)\right) .
\end{aligned}
$$

Then,

$$
\mathbf{E}\left(y_{x(B)}\right)=\int_{R} t d \mu(t), \mathbf{E}\left(y_{x\left(B_{i}\right)}\right)=\int_{R} t d \mu_{i}(t)
$$

Moreover,

$$
\begin{aligned}
\mu(D) & =\mathbf{m}(y(D \backslash\{0\}) \cdot x(B))=\mathbf{m}\left(y(D \backslash\{0\}) \cdot \bigoplus_{i=1}^{\infty} x\left(B_{i}\right)\right)= \\
& =\mathbf{m}\left(\bigoplus_{i=1}^{\infty}\left(y(D \backslash\{0\}) \cdot x\left(B_{i}\right)\right)\right)=\sum_{i=1}^{\infty} \mathbf{m}\left(y(D \backslash\{0\}) \cdot x\left(B_{i}\right)\right)= \\
& =\sum_{i=1}^{\infty} \mu_{i}(D) .
\end{aligned}
$$

Therefore we have

$$
\mathbf{E}\left(y_{x(B)}\right)=\int_{R} t d \mu(t)=\sum_{i=1}^{\infty} \int_{R} t d \mu_{i}(t)=\sum_{i=1}^{\infty} \mathbf{E}\left(y_{x\left(B_{i}\right)}\right) .
$$

Since $\mu(D)=\mathbf{m}(y(D \backslash\{0\}) \cdot x(B)) \leq \mathbf{m}(y(D))$, then we have

$$
\left|\mathbf{E}\left(y_{x(B)}\right)\right|=\left|\int_{R} t d \mu(t)\right| \leq \int_{R}|t| d \mu(t) \leq \int_{R}|t| d \mathbf{m}_{y}(t)<\infty
$$

Theorem 3. Let $x, y: \mathcal{B}(R) \rightarrow R$ be the IF-observables and $y$ be integrable, that is, there exists $\mathbf{E}(y)=\int_{R} t d \mathbf{m}_{y}(t)$. Then there exists a Borel measurable function $f: R \rightarrow R$ such that

$$
\mathbf{E}\left(y_{x(B)}\right)=\int_{B} f d \mathbf{m}_{x}
$$

for each $B \in \mathcal{B}(R)$.

Proof. Define $\mu: \mathcal{B}(R) \rightarrow[0,1]$ by the formula $\mu(B)=\mathbf{m}(x(B))=\mathbf{m}_{x}(B)$ and $v: \mathcal{B}(R) \rightarrow$ $[0,1]$ by the formula $v(B)=\mathbf{E}\left(y_{x(B)}\right)=\int_{R} t d \mathbf{m}_{y_{x(B)}}(t)$.

If $\mathbf{m}_{x}(B)=0$, that is, $\mathbf{m}(x(B))=0$, then

$$
\mathbf{m}\left(y_{x(B)}(D)\right)=\mathbf{m}\left(y(D \backslash\{0\} \cdot x(B)) \leq \mathbf{m}\left(\left(1_{\Omega}, 0_{\Omega}\right) \cdot x(B)\right)=\mathbf{m}(x(B))=0 .\right.
$$


Hence,

$$
v(B)=\mathbf{E}\left(y_{x(B)}\right)=\int_{R} t d \mathbf{m}_{y_{x(B)}}(t)=0 .
$$

Therefore $v \ll \mu$ and by Radom-Nikodym theorem there exists the Borel measurable function $f: R \rightarrow R$ such that

$$
\mathbf{E}\left(y_{x(B)}\right)=\int_{B} f d \mathbf{m}_{x}
$$

for each $B \in \mathcal{B}(R)$.

Now we are able to define a notion of a conditional intuitionistic fuzzy mean value (expectation).

Definition 9. If $x, y: \mathcal{B}(R) \rightarrow R$ are the IF-observables and $y$ is integrable, then the conditional IF-mean value (expectation) $\mathbf{E}(y \mid x)=f$ is the Borel measurable function such that

$$
\mathbf{E}\left(y_{x(B)}\right)=\int_{B} \mathbf{E}(y \mid x) d \mathbf{m}_{x}
$$

for each $B \in \mathcal{B}(R)$.

Now we show the connection between a conditional intuitionistic fuzzy mean value $\mathbf{E}(y \mid x)$ and a conditional intuitionistic probability $\mathbf{p}(\mathbf{A} \mid x)$ introduced by B. Riečan in [12] (see Remark 1). Recall that a conditional intuitionistic fuzzy probability is a Borel measurable function $f$ (i.e., $B \in \mathcal{B}(R) \Longrightarrow f^{-1}(B) \in \mathcal{B}(R)$ ) such that

$$
\int_{B} \mathbf{p}(\mathbf{A} \mid x) d \mathbf{m}_{x}=\mathbf{m}(\mathbf{A} \cdot x(B))
$$

for each $B \in \mathcal{B}(R)$, where $\mathbf{m}: \mathcal{F} \rightarrow[0,1]$ is the intuitionistic fuzzy state, $\mathbf{A} \in \mathcal{F}$ is an intuitionistic fuzzy event and $x: \mathcal{B}(R) \rightarrow \mathcal{F}$ is an intuitionistic fuzzy observable.

Remark 1. Take $\mathbf{A} \in \mathcal{F}$ and define the IF-observable $y_{\mathbf{A}}: \mathcal{B}(R) \rightarrow \mathcal{F}$ by

$$
y_{\mathbf{A}}(B)= \begin{cases}\left(0_{\Omega}, 1_{\Omega}\right), & \text { if } B=\varnothing \\ \mathbf{A}, & \text { if } B=\{1\} \\ y_{\mathbf{A}}(B \cap\{1\}), & \text { if } B \neq \varnothing, B \neq R, B \in \mathcal{B}(R) \\ \left(1_{\Omega}, 0_{\Omega}\right), & \text { if } B=R\end{cases}
$$

Then $\mathbf{E}\left(y_{\mathbf{A}} \mid x\right)=\mathbf{p}(\mathbf{A} \mid x)$ holds $\mathbf{m}_{x}$-almost everywhere.

Proof. Namely,

$$
\begin{aligned}
\int_{B} \mathbf{E}\left(y_{\mathbf{A}} \mid x\right) d \mathbf{m}_{x} & =\mathbf{E}\left(y_{\mathbf{A}} \cdot x(B)\right)=\int_{R} \operatorname{id} d \mathbf{m}\left(y_{\mathbf{A}} \cdot x(B)\right)=\int_{R} \mathrm{id} d \mathbf{m}(\mathbf{A} \cdot x(B))= \\
& =\mathbf{m}(\mathbf{A} \cdot x(B))=\int_{B} \mathbf{p}(\mathbf{A} \mid x) d \mathbf{m}_{x}
\end{aligned}
$$

for each $B \in \mathcal{B}(R)$.

Hence $\mathbf{E}\left(y_{\mathbf{A}} \mid x\right)=\mathbf{p}(\mathbf{A} \mid x)$ holds $\mathbf{m}_{x}$-almost everywhere.

\section{Conclusions}

This paper is concerned with the probability theory of intuitionistic fuzzy sets. We defined the indefinite integral for an intuitionistic fuzzy observable. We introduced the notion of a conditional intuitionistic fuzzy mean value and we showed a connection with 
the conditional intuitionistic fuzzy probability induced by an intuitionistic fuzzy state. We used a Kolmogorov approach and the notions from a classical probability theory for construction. Another way of obtaining the results is a construction of MV-algebra of intuitonistic fuzzy events and using the results from MV-algebras. Unfortunately, this approach leads to the crisp results as in [11], because the family of intuitionistic fuzzy events $\mathcal{F}$ does not contain a set $\neg \mathbf{A}=\left(1_{\Omega}-v_{A}, 1_{\Omega}-v_{A}\right)$. In future research we would like to prove the martingale convergence theorem for a conditional intuitionistic fuzzy mean value.

Funding: This research was funded by Mobility project BAS-SAS-21-01.

Institutional Review Board Statement: Not applicable.

Informed Consent Statement: Not applicable.

Data Availability Statement: Not applicable.

Conflicts of Interest: The author declares no conflict of interest.

\author{
Abbreviations \\ The following abbreviations are used in this manuscript: \\ IF Intuitionistic Fuzzy
}

\title{
References
}

1. De Korvin, A.; Kleyle, R. A note on fuzzy conditional expectation. Stoch. Anal. Appl. 1998, 16, 1005-1017. [CrossRef]

2. Näther, W.; Wünsche, A. On the conditional variance of fuzzy random variables. Metrika 2007, 65, 109-122. [CrossRef]

3. Tang, S.; He, Y. Conditional Value at Risk Methodology under Fuzzy-Stochastic approach. In Intelligent Computing Theories, Proceedings of the ICIC Conference, Nanning, China, 28-31 July 2013; Huang, D.S., Bevilacqua, V., Eds.; Lecture Notes in Computer Science; Springer: Berlin, Germany, 2013; Volume 7995, pp. 163-172.

4. You, C. On the Conditional Expected Value for Fuzzy Variables. In Proceedings of the Sixth International Conference on Business Intelligence and Financial Engineering, Hangzhou, China, 14-16 November 2013; Yu, L., Chen, R., Wang, S., Eds.; IEEE: Piscataway, NJ, USA, 2013; pp. 604-608.

5. Bertanha, M.; Imbens, G.W. External Validity in Fuzzy Regression Discontinuity Designs. J. Bus. Econ. Stat. 2020, 38, 593-612. [CrossRef]

6. Riečan, B. On the conditional expectation of observables in MV algebras of fuzzy sets. Fuzzy Sets Syst. 1999, 102, 445-450. [CrossRef]

7. Jurečková, M. On the conditional expectation on probability MV-algebras with product. Soft Comput. 2001, 5, 381-385. [CrossRef]

8. Atanassov, K.T. Intuitionistic fuzzy sets. VII ITKR Session, Sofia, 20-23 June 1983 (Deposed in Centr. Sci.-Techn. Library of the Bulg. Acad. of Sci., 1697/84). Repr. Int. Bioautom. 2016, 20, S1-S6. (In Bulgarian)

9. Zadeh, L.A. Fuzzy sets. Inf. Control 1965, 8, 338-358. [CrossRef]

10. Zadeh, L.A. Probability measures on fuzzy sets. J. Math. Anal. Appl. 1968, 23, 421-427. [CrossRef]

11. Valenčáková, V. A Note on the Conditional Expectation of IF-Observables. In Fuzzy Logic and Applications, Proceedings of the WILF Conference, Palermo, Italy, 9-12 June 2009; Di Gesú, V., Pal, S.K., Eds.; Lecture Notes in Computer Science; Springer: Berlin, Germany, 2009; Volume 5571, pp. 85-92.

12. Riečan, B. Analysis of fuzzy logic models. In Intelligent Systems; Koleshko, V., Ed.; INTECH: Rijeka, Croatia, 2012; pp. 219-244.

13. Atanassov, K.T. Intuitionistic Fuzzy Sets: Theory and Applications, 1st ed.; Physica Verlag: New York, NY, USA, $1999 ;$ pp. 1-137.

14. Atanassov, K.T. On Intuitionistic Fuzzy Sets Theory; Springer: Berlin, Germany, 2012; pp. 1-52.

15. Riečan, B. On the probability and random variables on IF events. In Applied Artifical Intelligence, Proceedings of the 7 th International FLINS Conference, Genova, Italy, 29-31 August 2006; Ruan, D., D’hondt, P., Eds.; World Scientific: Singapore, 2009 ; pp. 138-145.

16. Lendelová, K.; Riečan, B. Weak law of large numbers for IF-events. In Current Issues in Data and Knowledge Engineering; EXIT: Warsaw, Poland, 2004; pp. 309-314.

17. Lendelová, K. Conditional IF-probability. In Soft Methods for Integrated Uncertainty Modelling; Springer: Berlin/Heidelberg, Germany, 2006; Volume 37, pp. 275-283.

18. Čunderlíková, K. A note on mean value and dispersion of intuitionistic fuzzy events. Notes Intuitionistic Fuzzy Sets 2020, 26, 1-8. [CrossRef] 